OPEN ACCESS

Edited by:

Tetsuo Kida,

National Institute for Physiological

Sciences, Japan

Reviewed by:

Shane McKie,

University of Manchester, UK

Stephan Doering,

Medical University of Vienna, Austria

${ }^{*}$ Correspondence:

Karin Labek

karin.labek@uibk.ac.at

Received: 09 March 2016 Accepted: 22 June 2016 Published: 05 July 2016

Citation:

Labek K, Viviani R, Gizewski ER, Verius $M$ and Buchheim A (2016) Neural Correlates of the Appraisal of

Attachment Scenes in Healthy

Controls and Social Cognition-An

fMRI Study.

Front. Hum. Neurosci. 10:345. doi: 10.3389/fnhum.2016.00345

\section{Neural Correlates of the Appraisal of Attachment Scenes in Healthy Controls and Social Cognition- An fMRI Study}

\author{
Karin Labek ${ }^{1 *}$, Roberto Viviani ${ }^{1,2}$, Elke R. Gizewski ${ }^{3,4}$, Michael Verius ${ }^{3,4}$ \\ and Anna Buchheim ${ }^{1}$ \\ ${ }^{1}$ Department of Clinical Psychology, Institute of Psychology, University of Innsbruck, Innsbruck, Austria, ${ }^{2}$ Department \\ of Psychiatry and Psychotherapy III, University of UIm, UIm, Germany, ${ }^{3}$ Department of Neuroradiology, Medical University \\ Innsbruck, Innsbruck, Austria, ${ }^{4}$ Neuroimaging Research Core Facility, Innsbruck Medical University, Innsbruck, Austria
}

The human attachment system is activated in situations of danger such as potential separation, threats of loss of a significant other and potential insecurity on the availability of the attachment figure. To date, however, a precise characterization of the neural correlates of the attachment system in healthy individuals is lacking. This functional magnetic resonance imaging (fMRI) study aims at characterizing the distinctive neural substrates activated by the exposure to attachment vs. non-attachment scenes. Healthy participants $(N=25)$ were presented scenes from the Adult Attachment Projective Picture System (AAP), a validated set of standardized attachment-related pictures extended by a control picture stimulus set consisting of scenes without attachment-related content. When compared to the control neutral pictures, attachment scenes activated the inferior parietal lobes (IPLs), the middle temporal gyrus (MTG), and the anterior medial prefrontal cortex (mPFC). These areas are associated with reasoning about mental representations, semantic memory of social knowledge, and social cognition. This neural activation pattern confirms the distinctive quality of this stimulus set, and suggests its use as a potential neuroimaging probe to assess social cognition/mentalizing related to attachment in healthy and clinical populations.

Keywords: attachment, fMRI, social cognition, mentalizing, temporo-parietal-junction

\section{INTRODUCTION}

The human attachment system is a behavioral system that is responsible for triggering specific responses when situations arise that signal potential physical or psychological danger or stress. In this respect, the human attachment system differs from other related innate attachment systems, such as the caregiving, affiliative, and romantic/sexual systems. Attachment theory is rooted within an evolutionary context and may be viewed as one of the motivational systems that have the function to maximize the chances of survival. When activated, the attachment system motivates behaviors that aim at responding the child's need for protection or support, such as seeking the proximity of the caregiver (Bowlby, 1969, 1973, 1980).

Several neuroimaging studies have investigated the neural correlates of the caregiving (e.g., Bartels and Zeki, 2004; Leibenluft et al., 2004; Nitschke et al., 2004; Lenzi et al., 2009; Riem et al., 2012; Wittfoth-Schardt et al., 2012), the affiliative (e.g., Vrtička et al., 2008) and the romantic/sexual 
system (e.g., Bartels and Zeki, 2000, 2004; Gillath et al., 2005; Coan et al., 2006).

These studies have used various approaches to identify the neural correlates of distinctive attachment-related systems, such as pictures or video clips of one's own vs. unknown children, pictures of romantic partners, have one's hand held while being subjected to painful stimuli, or thinking about relationship scenarios. Notwithstanding the differences in methods and aims, several studies have reported a shared neural activity for the romantic and maternal attachment in regions associated with reward and motivation (ventral striatum, the putamen and the globus pallidus) and affective processing (orbitofrontal cortex).

However, much less is known about the neural correlates of the attachment system related to own representations. In the present study, we investigated the neural substrates activated by appraising situations that potentially activate the attachment system in the healthy adult.

In the child, the attachment system is activated on the one hand by attachment-related threats (for example, when infants are separated from their caregivers), and on the other hand by situations that are inherently threatening or increase the likelihood of danger (such as isolation, illness, injury; see Bowlby, $1969,1973,1980)$. In the adult, the attachment system may be activated by attachment-related stress, such as the loss of a loved one, and by a wider set of emotional or interpersonal situations. This activation is mediated by mental representations or schemata that encode past experiences of interactions with attachment figures in times of threat, of the availability or the intentions of attachment-figures, of the capacity of the attachment-figure to respond to our needs. Thus, according to attachment theory, in the adult responses to stimuli of social nature may also be organized by the attachment system when these stimuli are appraised through the appropriate schemata.

This fact is exploited by diagnostic instruments, such as the Adult Attachment Projective Picture System (AAP; George and West, 2001, 2012; Buchheim et al., 2006, 2008), that aim at characterizing in the adult the quality of the responses triggered by the activation of the attachment system. In the AAP interview, individuals are presented with scenes depicting attachment relationships or potential relationships in the presence of figural elements alluding to threatening conditions such as illness, separation, loneliness, or death. The quality of the attachmentrelated response of the individual is assessed through a semistructured interview that measures the individual's attachment representations activated by these scenes.

To characterize the neural substrates activated by the exposure to the attachment scenes of the AAP and that are involved in processing these stimuli we developed a new set of control pictures that depicted almost the identical scenes, except for the replacement of the threatening figural elements with others of a more neutral nature (for example, see Figure 1). In developing these control pictures we made every effort to create scenes portraying social interactions and in which individuals appeared as possessing a purpose and/or mental activity, as in the original AAP scenes. The function of these control pictures was not one of offering impoverished scenes of human interaction, or individuals without a discernible mental content; instead, we attempted to recreate scenic elements of similar complexity as in the original AAP set, but without the reference to the cues that may activate the attachment system. Activated neural correlates were sought that emerged in the comparison between the exposure to the attachment scenes of the AAP diagnostic interview and the modified neutral scenes as a control condition.

The present study belongs to the set of studies that investigate the neural correlates of the own representations of attachment with respect to attachment related threats or loss of the reference person and insecurity about its permanence or availability (Buchheim et al., 2006, 2008, 2012, 2013). To date, these studies have instructed the participants to tell narratives to the AAP pictures in the functional magnetic resonance imaging (fMRI) environment or they used individualized text passages from the AAP interview to activate the attachment system. These studies intended to characterize differences between healthy participants and patient groups, such as depressives or individuals suffering from borderline personality disorder at a neural level. While these studies have successfully identified differences between healthy controls and patients, it has been difficult to interpret the data univocally in the absence of an adequate control stimulus set. Therefore, the present study is also meant as a methodological development to achieve improved specificity in the detection of the substrates associated with the attachmentrelated cues.

One issue raised by these studies is the involvement of areas, such as the right temporo-parietal-junction (TPJ; Buchheim et al., 2008), which in other studies has been shown to be part of a network underlying social cognition, and is often activated in response to social inputs, like social interactions (Frith and Frith, 2003). In the studies of the human attachment system (Buchheim et al., 2008), these areas are more often active in the presence of scenes depicting two interacting individuals (dyadic scenes) than in scenes showing only one person (monadic scenes). In attachment theory, social cognition abilities are considered essential to interact suitably with the attachment figure. These abilities are needed to make appropriate attributions about the mental states of others that may explain their behavior in the interaction. Especially in times of need or in the presence of threats, what individuals know about the mental state of attachment figures is important to behave so as to obtain proximity and care (see Bowlby, 1969, 1973, 1980). Recruitment of brain areas associated with social cognition (e.g., temporal poles, TPJ, posterior superior temporal sulcus), has been reported in few other studies of attachment (Leibenluft et al., 2004). The more complex interpersonal nature/information of the dyadic scenes may indicate the need for greater cognitive processing and could be particularly effective in eliciting schematic representations of social interactions.

A further issue arising from the comparison with studies of different forms of attachment is the predominantly negative valence of stimuli activating the human attachment system. This contrasts with the generally appetitive nature of stimuli activating the affiliative, caregiving, and romantic systems. Processing of 
stimuli of negative emotional valence is often associated with the activation of the amygdala (Davis and Whalen, 2001; Adolphs et al., 2005). This activation, however, may be related to the generally high arousing nature of these stimuli (Adolphs, 2010). Strathearn and Kim (2013) reported amygdala responses to positively or negatively valenced cues (own vs. unknown child, happy, sad, neutral faces) modulated by personal relevance. To address this issue, we characterized the amount of negative valence and arousal in our stimulus set relative to other sets commonly used in neuroimaging studies, and examined the activation of the amygdala with region-of-interest (ROI) analyses.

\section{MATERIALS AND METHODS}

The study protocol was approved by the Ethical Committee of the Medical University of Innsbruck and was in compliance with National legislation, the principles expressed in the Declaration of Helsinki, and the Code of Ethical Principles for Medical Research Involving Human Subjects of the World Medical Association. All participants gave written informed consent.

\section{Participants}

A sample of $N=25$ healthy volunteers were recruited at LeopoldFranzens University in Innsbruck (age mean/SD 22.7/1.8, age range: 19-26, 12 females, see Table 1). Depressiveness was measured with a computerized German Version of the Centre for Epidemiologic Studies Depression Scale (CES-D, Radloff, 1977; German Version: Hautzinger and Bailer, 1993). The present and long-lasting anxiety levels were assessed by the State-Trait Anxiety Inventory (STAI, Spielberger et al., 1970; German Version: Laux et al., 1981) All participants had scores in a subclinical range. Finally participants were assessed with the International Neuropsychiatric Interview (M.I.N.I. 5.0.0,
TABLE 1 | Participants.

\begin{tabular}{lc}
\hline & $\boldsymbol{n}=\mathbf{2 5}$ \\
\hline Age mean (std. dev.) & $22.7(1.8)$ \\
Female (\%) & $12(48)$ \\
Male (\%) & $13(52)$ \\
ADS (std. dev.) & $10.08(10.39)$ \\
STAl-S (std. dev.) & $35.60(11.55)$ \\
STAl-T (std. dev.) & $35.68(11.36)$
\end{tabular}

Sheehan et al., 1998, German version) to exclude previous psychiatric or psychological illnesses.

\section{Stimulus Material}

The stimulus material consisted of attachment-related pictures $\left(\mathrm{AAP}^{\odot}\right.$ George and West, 2012) and their modified neutral counterparts as a control condition. The AAP, an established and validated interview to assess attachment representations, is comprised of eight black and white line drawn pictures: seven attachment-related drawings and one neutral scene used as a warm-up. The attachment-related pictures are designed to activate the attachment system and to elicit mental engagement with attachment-related experiences (such as loneliness, helplessness, loss following illness, separation, solitude, death, and abuse). These drawings contain no facial expressions, and figures are drawn in summary strokes without precise details, but include the essential characteristics to recognize the attachment context. Drawings portray adult-adult dyads, adult-child dyads, adults alone, and children alone and capture attachment across the life span, infancy to old age.

For the control condition, pictures of attachment scenes from the AAP (George and West, 2012) were expanded with a set of carefully matched control scenes of nonattachment "neutral" character (Figure 1). The control battery consisted of eight line drawings of non-attachment pictures developed by the first author. In the control set, allusions to
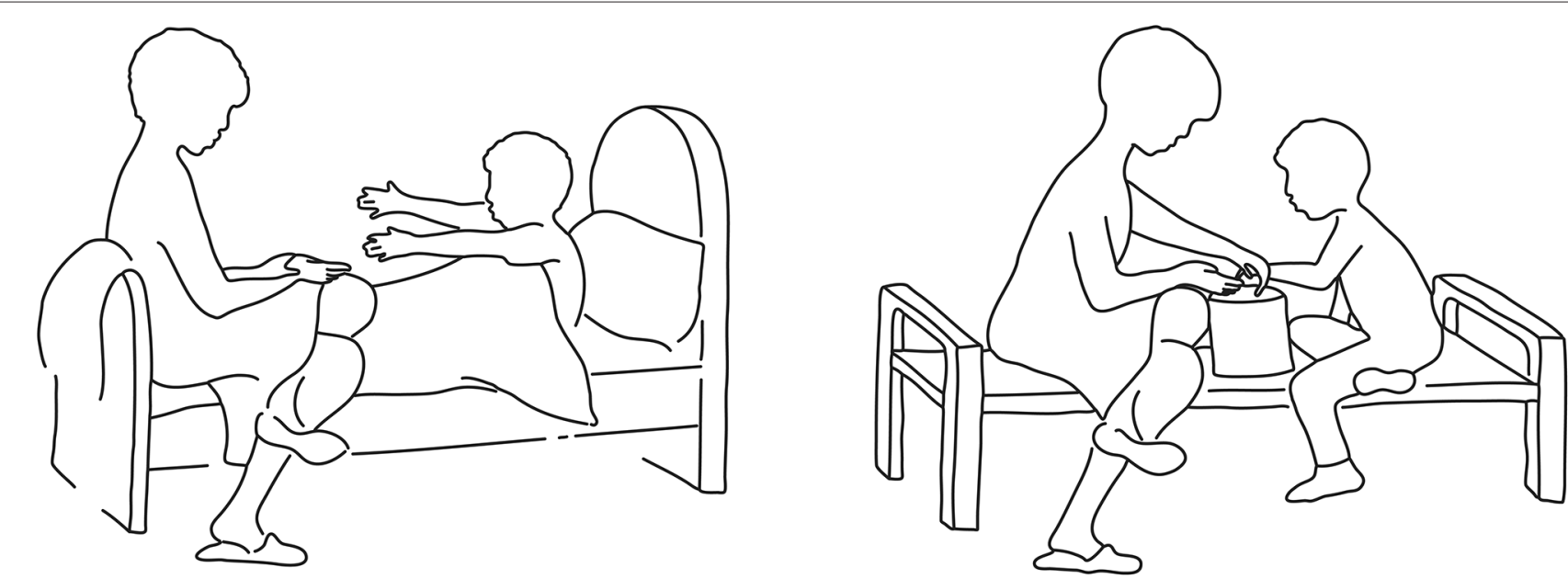

FIGURE 1 | Example of an original attachment-related scene of the Adult Attachment Projective Picture System (AAP) (left) and the newly created corresponding control scene (right). 
loneliness, potential rejection, death or illness, etc., were removed while replicating objects/characters, spatial structure and object configuration as closely as possible. Therefore, elements such as the monadic or dyadic nature of the scene, the age of the individuals depicted in it, etc., was precisely matched in the control picture set. The control scenes had the same size and were aligned with the corresponding AAP pictures. Care was given to prepare all pictures, attachment-related and nonattachment related, with the same stroke width, thickness, and size to make them formally identical. Since the original AAP warm-up scene contains no attachment-related content, we created a matched attachment-related scene (the picture of two children playing ball was transformed into a scene of potential separation). In total, there were 16 pictures, eight for each condition.

\section{Validation of the Stimulus Material}

To validate the stimulus set, a preliminary behavioral study was conducted on a separate group of 97 healthy subjects (age mean/SD 23.15/5.12, 72 females). In this preliminary study, all scenes (attachment-related and control) were rated according to the dimensions valence and arousal through the self-assessment manikin (SAM) scales developed by Bradley and Lang (1994). This pictorial rating system records self-assessments of experienced emotion, rated according to icons depicting a 5-point scale along the dimensions affective valence and arousal. This rating system was developed to assess sets of standardized stimuli for experimental investigations that are widely used in neuroimaging investigations of emotion (International Affective Picture System [IAPS], Lang et al., 2008). Hence, the collection of these validation data makes it possible to compare the present stimulus set with other visual stimulus sets used in the neuroimaging literature.

Valence ratings of the attachment-related pictures (mean score $=1.85, \mathrm{SD}=1.00$ ) were significantly more negative in comparison to the ratings of neutral pictures (mean score $=3.75$, $\mathrm{SD}=0.98 ; T=-37.971, p<0.000)$. Attachment-related pictures were rated of higher arousal (mean score $=3.06, \mathrm{SD}=1.18$ ) than neutral pictures (mean score $=2.28, \mathrm{SD}=1.08 ; T=13.577$, $p<0.000$; Knoll, 2015). These results confirmed that the new control picture-set significantly differed from the original AAP-set. However, it also suggested that the attachment-related pictures differed from typical negatively valenced visual stimuli used in previous neuroimaging studies. In those studies, the arousal of negatively valenced scenes may be higher than the arousal of the attachment-related scenes in the AAP set. Figure 2 shows the combination of the arousal and valence scores of the pictures in our set, plotted in a two-dimensional affective space representing the valence-arousal relationship. The gray dots show the arousal and valence of the IAPS, which is the source of material often used in neuroimaging studies (Lang et al., 2008). This plot shows the association of extreme valence scores (either positive or negative) with high arousal scores in the IAPS. In our picture set, negative emotional scores (in red) were not associated with arousal values substantially above the middle line. As in the IAPS set, there was an association of degree of negative valence in the attachment-related pictures with the

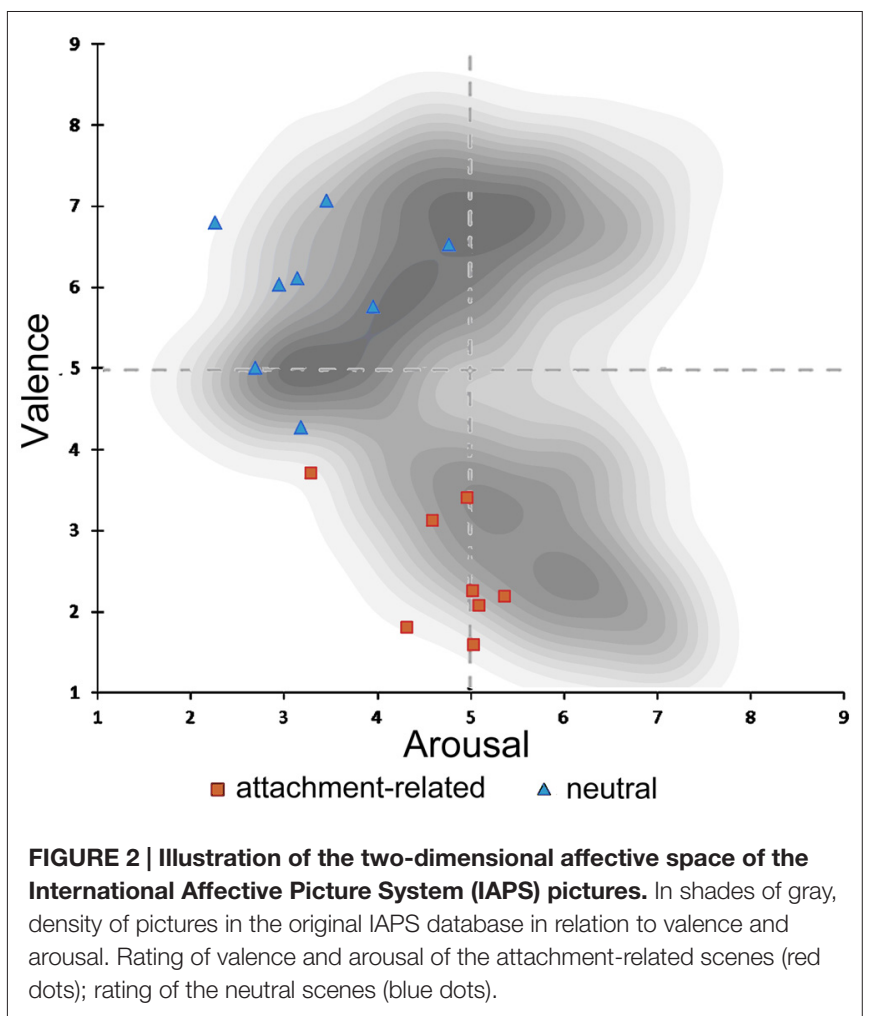

level of arousal; however, the arousal levels were consistently less than what one would expect for pictures of comparable negative valence.

\section{fMRI Data Acquisition, Preprocessing, and Statistical Modelling}

Participants were placed supine in the scanner, wearing earplugs to muffle noise. Head fixation was assured through a foam rubber device mounted on the head coil. Data were acquired using a T2*-weighted echo planar imaging (EPI) sequence on a $3 \mathrm{~T}$ Siemens Verio scanner (repetition time (TR)/echo time (TE) 2900/30 ms, transversal acquisitions, phase encoding anteriorposterior, field of view (FOV) $1260 * 1260$, slice thickness $2.5 \mathrm{~mm}$ with a gap of $1 \mathrm{~mm}$, giving a voxel size of $1.88 \times 1.88 \times 3.5 \mathrm{~mm}$, flip angle $90^{\circ}$, pixel bandwidth $1680 \mathrm{~Hz}$ ). Data were acquired in two separate runs, each of which contained eight trials in which a scene was shown for $15 \mathrm{~s}$, followed by a fixation cross for further $15 \mathrm{~s}$ Participants were instructed to pay attention to the persons depicted in scenes. Each run contained eight trials, equally divided into two of the two types of scenes (attachmentrelated or control). Scenes constituting the control version of the original attachment-related AAP scenes were shown in different runs, so that each run contained exposure to the whole set of eight scenes (attachment-related or control). To avoid systematic effects, the sequence in which scenes were recruited in the two runs was systematically changed across subjects.

Data were analyzed after realignment, normalization (resampling size, $2 \mathrm{~mm}$ isotropic) and smoothing $(8 \mathrm{~mm}$ full width at half maximum (FWHM)) with the software package 
SPM8 ${ }^{1}$. Contrasts of interest were estimated at the first level within each participant separately, and brought to the second level to account for the random effect of subjects. We report effects at $p<0.05$, cluster-level corrected, with clusters defined by the threshold $p<0.001$, uncorrected.

The data were analyzed with a model in which the blocks during which pictures were displayed were convolved with a standard blood oxygen level dependent (BOLD) hemodynamic function to account for the delay of onset of the signal. A design matrix was constructed containing two factors of two levels each: attachment/control and monadic/dyadic. Appropriate contrasts were taken to the second level to estimate the effects of

${ }^{1}$ http://www.fil.ion.ucl.ac.uk/spm/ attachment and its interaction with the monadic or dyadic nature of the pictures.

\section{RESULTS}

In the contrast attachment vs. neutral we found a statistical significant activation in the left inferior parietal lobe (IPL)/temporo-parietal junction (TPJ) (MNI coordinates $x$, $y, z=-46,-52,42, t=6.67$, number of voxels in cluster $k=833, p<0.001$, cluster-level corrected), in the left middle temporal gyrus (MTG) $(x, y, z=-66,-24,-12, t=5.10$, $k=258, p=0.005)$. These clusters were accompanied by less pronounced activations on the opposite side, which failed to reach significance after correction (IPL: $x, y, z=62,-54,36$,
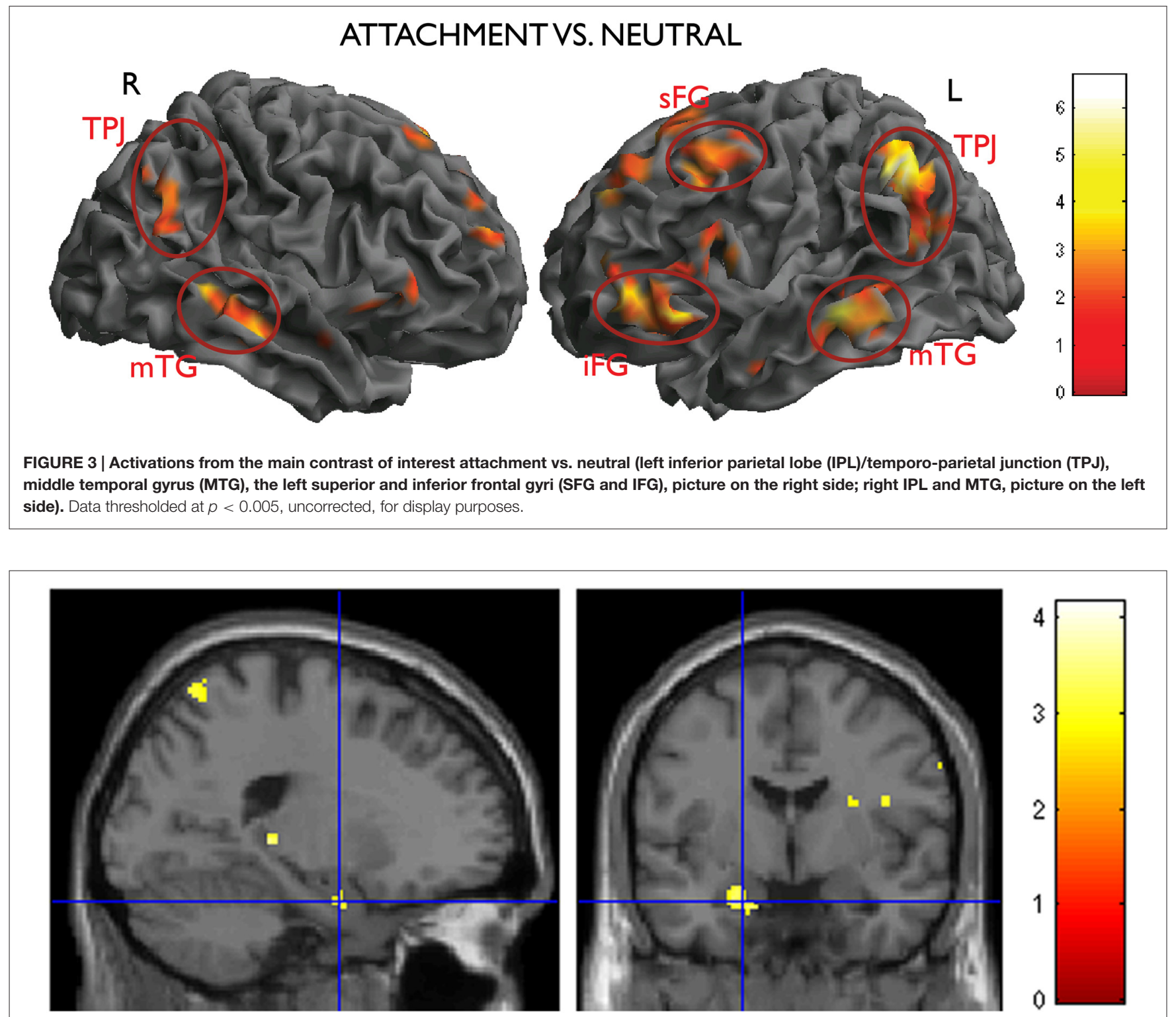

FIGURE 4 | Deactivation of the amygdala in the main contrast attachment vs. neutral pictures. Same display threshold as in the previous Figure ( $p<0.005)$. 
$t=4.69, k=54$, n.s.; MTG, $x, y, z=52,-20,-16, t=4.98$, $k=64$, n.s.). In the prefrontal lobes, the left superior and inferior frontal gyri (SFG and IFG) and the anterior medial prefrontal cortex ( $\mathrm{mPFC})$ were also activated in this contrast $(x, y, z=-42$, $16,56, t=5.40, k=273, p=0.004 ; x, y, z$; Figure 3).

A question of interest was the involvement of the amygdala, given the combination of marked negative emotional valence and low arousal values of these pictures demonstrated by the behavioral validation study (see Figure 2 in the "Materials and Methods" Section). There was a bilateral light deactivation of the amygdala in the contrast attachment vs. neutral, which however was not significant after correction $(x, y, z=-24$, $-2,-24, t=-3.55 ; x, y, z=22,0,-22, t=-2.54$; Figure 4).

A second contrast of interest investigated the modulation of the effect of attachment by type of scenes (dyadic or monadic). In the contrast attach vs. control within the dyadic scenes group, the right temporo-parietal region was activated more strongly (effect within dyadic scenes: $x, y, z=56,-52,34, t=5.75, k=517$, $p<0.001$; effect within monadic scenes: $x, y, z=54,-64,40$, $t=3.84, k=19$, n.s.; interaction: $x, y, z=62,-32,16, t=4.33$, $k=47$, n.s; see Figure 3, top row). An interaction between the two factors attachment/neutral $\times$ dyadic/monadic was observed in the occipital lobes bilaterally $(x, y, z=48,-78,6, t=5.44$, $k=123, p=0.079 ; x, y, z=-40,-80,-6, t=4.63, k=139$, $p=0.050)$.

There was no significant interaction in the opposite direction, i.e., for effects of attachment that were larger in the monadic than in the dyadic group. As one may see from Figure 5 (bottom row), this was due to effects of similar intensity within both picture types in the areas activated by attachment, such as the TPJ on the left. Also the apparent higher activation in the left IFG in the monadic group, visible in the bottom row of Figure 3, failed to achieve significance $(x, y, z=-36,38,2, t=3.19)$.

\section{DISCUSSION}

The main contrast of interest of our study revealed the activation of areas of predominantly associative nature, such as the IPL and the MTG, in association with attachment-related stimuli. These findings replicate those of previous studies in which the AAP pictures were used in comparisons between healthy participants and patients (Buchheim et al., 2006, 2008). Activations in the anterior insula have a similar interpretation but have been more explicitly associated with emotional representations ( $\mathrm{Gu}$ et al., 2013). This pattern of activation contrasts with those commonly obtained in studies of negatively valenced visual stimuli, which are characteristically more arousing and activate limbic structures such as the amygdala (for a quantitative review, see Costafreda et al., 2008). The activation of these limbic structures has been shown to be related to the early automatic processing of sensory aspects of the stimulus (Morris et al., 1999), which trigger arousal levels favoring its attentional prioritization for further processing (Vuilleumier, 2005). The lack of recruitment of these circuits in the present study suggests that appraisal of attachment content recruited processes that differed from those typically associated with the exposure to negatively valenced stimuli in many previous neuroimaging studies. Activation of the amygdala in studies of attachment styles that used facial expression stimuli may have detected differences in arousal and emotional reactivity (Vrtička et al., 2008).

The most prominent among the association areas activated by the attachment-related content was the TPJ, a region that numerous previous studies have identified as a neural correlate of theory of mind tasks and social cognition (for reviews, see Gallagher and Frith, 2003; Saxe and Kanwisher, 2003; Decety and Lamm, 2007; Van Overwalle, 2009; Saxe, 2010; Carter and Huettel, 2013). Theory of mind is a construct that refers to the cognitive capacity to form a representation of other people's beliefs and intentions (Premack and Woodruff, 1978; Baron-Cohen, 1997). Neuroimaging studies have investigated this construct through story comprehension tasks that require the correct attribution of beliefs after changes in the environment (Mar, 2011). Social cognition is a label that more generically refers to the adequate perception and evaluation of social stimuli and the processing of information about social interactions, including their affective components. Functional neuroimaging studies have associated social cognition processes with an extensive network of areas, which, beside the TPJ, also includes the middle temporal sulcus, the temporal poles, and the mPFC (Frith and Frith, 2012).

Neuroimaging studies of theory of mind have reported that left TPJ activations are more common when processing different subjective perspectives, whereas the right parietal junction was more specifically activated by processing states like beliefs (Perner et al., 2006). In the present study, dyadic pictures activated the TPJ on both sides, while monadic pictures activated this structure only on the left. This result is consistent with a more complex representation of beliefs and mental states in the presence of interactions between participants in the scenes, relative to pictures where the mental state of the individual in the scene could be inferred directly from pictorial elements such as her body posture (Peelen and Downing, 2007).

In attachment theory, the concept of mentalization refers to the capacity of the individual to understand one's own and other people's actions in the light of intentions, personal desires, needs or beliefs (Fonagy and Target, 2005). To predict behavior on the basis of mental states, the current literature divide mentalization processes in emotional and cognitive aspects. Emotional mentalizing abilities are referred to the ability to empathize emotionally like representations of intentions and believes. Emotional contagion/mentalization processes are thought to involve a network of brain area e.g., IFG and the IFL (Shamay-Tsoory, 2011) while cognitive mentalization processes activate brain regions like e.g., the TPJ (Saxe, 2006; Vrtička and Vuilleumier, 2012) or superior temporal sulcus (Frith and Frith, 2003). In the AAP interview, participants are invited to develop narratives over the motives, feelings and thoughts of the individuals depicted in the scenes. Activation of the neural substrates of social cognition in the present study suggests that the healthy participants in 


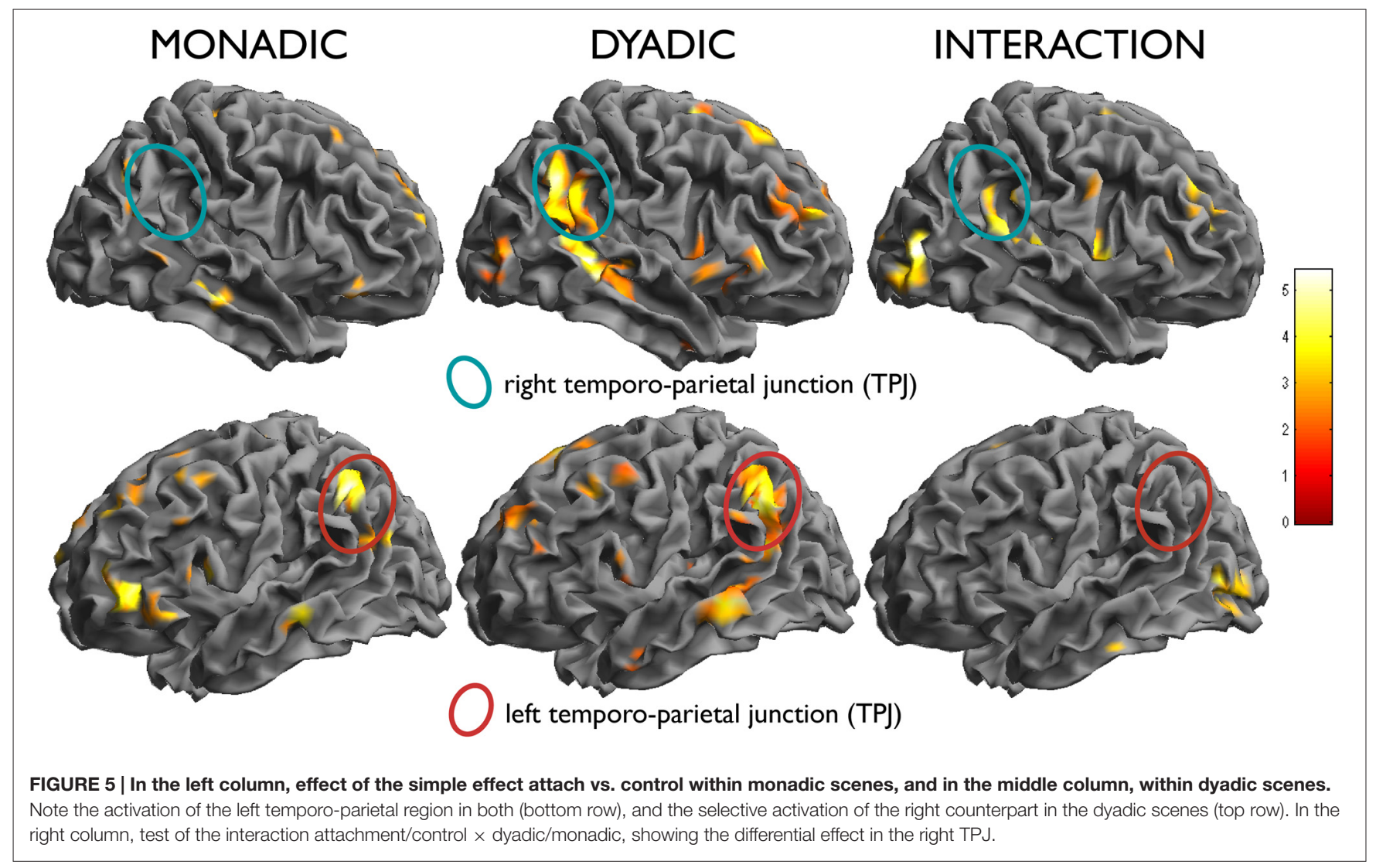

our sample spontaneously reacted to attachment scenes by elaborating such motives, especially in the presence of dyadic scenes.

Mentalization is an important individual trait that maybe associated with better affect- and self-regulation capacity (Fonagy and Luyten, 2009; Viviani et al., 2011). Dysfunction of self-regulation is crucial especially in the context of social relationships (Posner et al., 2002) Mentalization capacities in the adult are considered to develop from the internalization in childhood of the capacity for containment of the attachment figure. This containment enables the child to experience its own emotions without being overwhelmed by them (Fonagy et al., 1995; Bateman and Fonagy, 2004). In the theory underlying the AAI interview, this capacity is referred to as "reflective functioning" (Fonagy et al., 1998). Neuroimaging studies attempting to locate the neural correlates of mentalization have reported activations in the left MTG and IFG, two foci that were active also in the present study (Nolte et al., 2012).

In the alternative DSM-5 model for personality disorders (see "Results" Section), personality functioning (Criterion A) was included to assess the level of functional impairment associated with personality disorders (DSM-5; American Psychiatric Association, 2013). The level of personality functioning scale (LPFS; Bender et al., 2011) conceptualizes intrapersonal (identity, self-direction) and interpersonal (empathy, intimacy) components of the underlying personality structure. Hence, the ability to understand oneself and the social and internal world of others in terms of mental states are viewed as an essential trait of personality disorders. Here, working models of attachment theory and implicit representations of others represent a key theoretical and empirical issue (Skodol et al., 2011). Reflecting the increasing recognition of the importance of personality functioning, fMRI studies are beginning to apply assays eliciting activation in areas associated with representations of self (Doering et al., 2012) and empathy (Dziobek et al., 2008; Fan et al., 2011; Decety et al., 2013; Mier et al., 2013) to assess functional differences in personality disorders.

In summary, the present study provided evidence that exposure to the attachment scenes activated a cortical network that is recruited to represent the mental states of oneself and of others. These results are consistent with attachment theory, according to which the capacity to correctly interpret and process social cues from the attachment figure is important for responding appropriately in attachment relationships, thus ensuring security and proximity to the attachment figure. Our results suggest that while exposure to attachmentrelated themes, especially depicting interactions, specific processing takes place in brain areas associated with social cognition. The recruitment of social cognition-related areas while assessing attachment related pictures may be used in future studies to further explore its potential in detecting individual differences in mentalization capacities. By comparing 
the results of this and other functional assays, fMRI may contribute to clarifying the constructs involved in personality functioning.

\section{AUTHOR CONTRIBUTIONS}

The study was conceptualized by KL, AB, RV. The study setup and data collection were organized and conducted by KL, RV, ERG, MV. fMRI analyses was performed by KL and RV. KL and RV performed the statistical data analysis and contributed substantially to the result interpretation. AB and ERG provided

\section{REFERENCES}

Adolphs, R. (2010). What does the amygdala contribute to social cognition? Ann. N Y Acad. Sci. 1191, 42-61. doi: 10.1111/j.1749-6632.2010.05445.x

Adolphs, R., Gosselin, F., Buchanan, T. W., Tranel, D., Schyns, P., and Damasio, A. R. (2005). A mechanism for impaired fear recognition after amygdala damage. Nature 433, 68-72. doi: 10.1038/nature03086

American Psychiatric Association. (2013). Diagnostic and Statistical Manual of Mental Disorders: DSM-5. 5th Edn. Arlington, VA: American Psychiatric Association.

Baron-Cohen, S. (1997). Mindblindness: An Essay on Autism and Theory of Mind. Cambridge, MA: MIT press.

Bartels, A., and Zeki, S. (2000). The neural basis of romantic love. Neuroreport 11, 3829-3834. doi: 10.1097/00001756-200011270-00046

Bartels, A., and Zeki, S. (2004). The neural correlates of maternal and romantic love. Neuroimage 21, 1155-1166. doi: 10.1016/j.neuroimage.2003.11.003

Bateman, A. W., and Fonagy, P. (2004). Psychotherapy for Borderline Personality Disorder: Mentalisation-Based Treatment. Oxford: Oxford University Press.

Bender, D. S., Morey, L. C., and Skodol, A. E. (2011). Toward a model for assessing level of personality functioning in DSM-5, part I: a review of theory and methods. J. Pers. Assess. 93, 332-346. doi: 10.1080/00223891.2011.583808

Bowlby, J. (1969). Attachment. New York, NY: Basic Books.

Bowlby, J. (1973). Separation: Anxiety and Anger. New York, NY: Basic Books.

Bowlby, J. (1980). Loss. New York, NY: Basic Books.

Bradley, M. M., and Lang, P. J. (1994). Measuring emotion: the self-assessment manikin and the semantic differential. J. Behav. Ther. Exp. Psychiatry 25, 49-59. doi: 10.1016/0005-7916(94)90063-9

Buchheim, A., Erk, S., George, C., Kächele, H., Martius, P., Pokorny, D., et al. (2008). Neural correlates of attachment dysregulation in borderline personality disorder using functional magnetic resonance imaging. Psychiatry Res. 163, 223-235. doi: 10.1016/j.pscychresns.2007.07.001

Buchheim, A., Erk, S., George, C., Kächele, H., Ruchsow, M., Spitzer, M., et al. (2006). Measuring attachment representation in an fMRI environment: a pilot study. Psychopathology 39, 144-152. doi: 10.1159/000091800

Buchheim, A., Labek, K., Walter, S., and Viviani, R. (2013). A clinical case study of a psychoanalytic psychotherapy monitored with functional neuroimaging. Front. Hum. Neurosci. 7:677. doi: 10.3389/fnhum.2013.00677

Buchheim, A., Viviani, R., Kessler, H., Kächele, H., Cierpka, M., Roth, G., et al. (2012). Changes in prefrontal-limbic function in major depression after 15 months of long-term psychotherapy. PLoS One 7:e33745. doi: 10. 1371/journal.pone.0033745

Carter, R. M., and Huettel, S. A. (2013). A nexus model of the temporal-parietal junction. Trends Cogn. Sci. 17, 328-336. doi: 10.1016/j.tics.2013.05.007

Coan, J. A., Schaefer, H. S., and Davidson, R. J. (2006). Lending a hand social regulation of the neural response to threat. Psychol. Sci. 17, 1032-1039. doi: 10. 1111/j.1467-9280.2006.01832.x

Costafreda, S. G., Brammer, M. J., David, A. S., and Fu, C. H. (2008). Predictors of amygdala activation during the processing of emotional stimuli: a metaanalysis of 385 PET and fMRI studies. Brain Res. Rev. 58, 57-70. doi: 10.1016/j. brainresrev.2007.10.012

Davis, M., and Whalen, P. J. (2001). The amygdala: vigilance and emotion. Mol. Psychiatry 6, 13-34. doi: 10.1038/sj.mp.4000812 important intellectual contribution in commenting and revising the manuscript. KL, RV, and $\mathrm{AB}$ wrote the manuscript and edited its final version.

\section{ACKNOWLEDGMENTS}

The study was in part funded by a grant UNI-0404/1514 awarded to Karin Labek of the Tiroler Wissenschaftsfond (TWF, application "Neuronale Korrelate bei der Betrachtung bindungsrelevanter Szenen bei gesunden ProbandInnen", Nov. 2013) whose generous support is gratefully acknowledged.

Decety, J., Chen, C., Harenski, C., and Kiehl, K. A. (2013). An fMRI study of affective perspective taking in individuals with psychopathy: imagining another in pain does not evoke empathy. Front. Hum. Neurosci. 7:489. doi: 10. 3389/fnhum.2013.00489

Decety, J., and Lamm, C. (2007). The role of the right temporoparietal junction in social interaction: how low-level computational processes contribute to meta-cognition. The Neuroscientist 13, 580-593. doi: 10.1177/1073858407 304654

Doering, S., Enzi, B., Faber, C., Hinrichs, J., Bahmer, J., and Northoff, G. (2012). Personality functioning and the cortical midline structures-an exploratory fMRI study. PLoS One 7:e49956. doi: 10.1371/journal.pone.0049956

Dziobek, I., Rogers, K., Fleck, S., Bahnemann, M., Heekeren, H. R., Wolf, O. T., et al. (2008). Dissociation of cognitive and emotional empathy in adults with Asperger syndrome using the Multifaceted Empathy Test (MET). J. Autism Dev. Disord. 38, 464-473. doi: 10.1007/s10803-007-0486-x

Fan, Y., Wonneberger, C., Enzi, B., de Greck, M., Ulrich, C., Tempelmann, C., et al. (2011). The narcissistic self and its psychological and neural correlates: an exploratory fMRI study. Psychol. Med. 41, 1641-1650. doi: 10. 1017/S003329171000228X

Fonagy, P., Leigh, T., Kennedy, R., Mattoon, G., Steele, H., Target, M., et al. (1995). "Attachment, borderline states and the representation of emotions and cognition in self and other," in Rochester Symposium on Developmental Psychopathology: Emotion and Cognition, eds D. Cicchetti and S. T. Rochester (New York, NY: University of Rochester Press), 371-414.

Fonagy, P., and Luyten, P. (2009). A developmental, mentalization-based approach to the understanding and treatment of borderline personality disorder. Dev. Psychopathol. 21, 1355-1381. doi: 10.1017/S0954579409990198

Fonagy, P., and Target, M. (2005). Bridging the transmission gap: an end to an important mystery of attachment research? Attach. Hum. Behav. 7, 333-343. doi: 10.1080/14616730500269278

Fonagy, P., Target, M., Steele, H., and Steele, M. (1998). Reflective-Functioning Manual, Version 5.0, for Application to Adult Attachment Interviews. London: University College London.

Frith, U., and Frith, C. D. (2003). Development and neurophysiology of mentalizing. Philos. Trans. R. Soc. Lond. B Biol. Sci. 358, 459-473. doi: 10. 1098/rstb.2002.1218

Frith, C. D., and Frith, U. (2012). Mechanisms of social cognition. Annu. Rev. Psychol. 63, 287-313. doi: 10.1146/annurev-psych-120710-100449

Gallagher, H. L., and Frith, C. D. (2003). Functional imaging of 'theory of mind'. Trends Cogn. Sci. 7, 77-83. doi: 10.1016/s1364-6613(02)00025-6

George, C., and West, M. (2001). The development and preliminary validation of a new measure of adult attachment: the adult attachment projective. Attach. Hum. Dev. 3, 30-61. doi: 10.1080/14616730010024771

George, C., and West, M. (2012). The Adult Attachment Projective Picture System. New York, NY: Guilford Press.

Gillath, O., Bunge, S. A., Shaver, P. R., Wendelken, C., and Mikulincer, M. (2005). Attachment-style differences in the ability to suppress negative thoughts: exploring the neural correlates. Neuroimage 28, 835-847. doi: 10.1016/j. neuroimage.2005.06.048

Gu, X., Hof, P. R., Friston, K. J., and Fan, J. (2013). Anterior insular cortex and emotional awareness. J. Comp. Neurol. 521, 3371-3388. doi: 10.1002/cne. 23368 
Hautzinger, M., and Bailer, M. (1993). Allgemeine Depressionsskala (ADS). Weinheim: Beltz.

Knoll, A. (2015). Validierung Des Bildmaterials Des Adult Attachment Projective Picture Systems (AAP) Und Neu Gezeichneter Kontrollbilder Anhand Des Self-Assessment Manikins (SAM). Universität Innsbruck: Unveröffentlichte Masterarbeit.

Lang, P. J., Bradley, M. M., and Cuthbert, B. N. (2008). International Affective Picture System (IAPS): Affective Ratings of Pictures and Instruction Manual. Technical Report A-8. Gainesville, FL: University of Florida.

Laux, L., Glanzmann, P., Schaffner, P., and Spielberger, C. H. D. (1981). Das StateTrait-Angstinventar (STAI). Manual Weinheim: Beltz.

Leibenluft, E., Gobbini, M. I., Harrison, T., and Haxby, J. V. (2004). Mothers' neural activation in response to pictures of their children and other children. Biol. Psychiatry 56, 225-232. doi: 10.1016/j.biopsych.2004.05.017

Lenzi, D., Trentini, C., Pantano, P., Macaluso, E., Iacoboni, M., Lenzi, G. L., et al. (2009). Neural basis of maternal communication and emotional expression processing during infant preverbal stage. Cereb. Cortex 19, 1124-1133. doi: 10. 1093/cercor/bhn153

Mar, R. A. (2011). The neural bases of social cognition and story comprehension. Annu. Rev. Psychol. 62, 103-134. doi: 10.1146/annurev-psych-120709-145406

Mier, D., Lis, S., Esslinger, C., Sauer, C., Hagenhoff, M., Ulferts, J., et al. (2013). Neuronal correlates of social cognition in borderline personality disorder. Soc. Cogn. Affect. Neurosci. 8, 531-537. doi: 10.1093/scan/nss028

Morris, J. S., Öhman, A., and Dolan, R. J. (1999). A subcortical pathway to the right amygdala mediating "unseen" fear. Proc. Natl. Acad. Sci. U S A 96, 1680-1685. doi: 10.1073/pnas.96.4.1680

Nitschke, J. B., Nelson, E. E., Rusch, B. D., Fox, A. S., Oakes, T. R., and Davidson, R. J. (2004). Orbitofrontal cortex tracks positive mood in mothers viewing pictures of their newborn infants. Neuroimage 21, 583-592. doi: 10. 1016/j.neuroimage.2003.10.005

Nolte, T., Bolling, D. Z., Hudac, C. M., Fonagy, P., Mayes, L., and Pelphrey, K. A. (2012). Brain mechanisms underlying the impact of attachment-related stress on social cognition. Front. Hum. Neurosci. 7, 816-816. doi: 10.3389/fnhum. 2013.00816

Peelen, M. V., and Downing, P. E. (2007). The neural basis of visual body perception. Nat. Rev. Neurosci. 8, 636-648. doi: 10.1038/nrn2195

Perner, J., Aichhorn, M., Kronbichler, M., Staffen, W., and Ladurner, G. (2006). Thinking of mental and other representations: the roles of left and right temporo-parietal junction. Soc. Neurosci. 1, 245-258. doi: 10.1080/174709106 00989896

Posner, M. I., Rothbart, M. K., Vizueta, N., Levy, K. N., Evans, D. E., Thomas, K. M., et al. (2002). Attentional mechanisms of borderline personality disorder. Proc. Natl. Acad. Sci. U S A 99, 16366-16370. doi: 10.1073/pnas. 252644699

Premack, D., and Woodruff, G. (1978). Does the chimpanzee have a theory of mind? Behav. Brain Sci. 1, 515-526. doi: 10.1017/s0140525x00076512

Radloff, L. S. (1977). The CES-D scale: a self-report depression scale for research in the general population. Appl. Psychol. Meas. 1, 385-401. doi: 10.1177/ 014662167700100306

Riem, M. M. E., Bakermans-Kranenburg, M. J., van IJzendoorn, M. H., Out, D., and Rombouts, S. A. R. B. (2012). Attachment in the brain: adult attachment representations predict amygdala and behavioral responses to infant crying. Attach. Hum. Dev. 14, 533-551. doi: 10.1080/14616734.2012. 727252
Saxe, R. (2006). Uniquely human social cognition. Curr. Opin. Neurobiol. 16, 235-239. doi: 10.1016/j.conb.2006.03.001

Saxe, R. (2010). "The right temporo-parietal junction: a specific brain region for thinking about thoughts," in Handbook of Theory of Mind, eds A. Leslie and T. German (Philadelphia, PA: Psychology Press), 1-35.

Saxe, R., and Kanwisher, N. (2003). People thinking about thinking people: the role of the temporo-parietal junction in "theory of mind". Neuroimage 19, 1835-1842. doi: 10.1016/s1053-8119(03)00230-1

Shamay-Tsoory, S. G. (2011). The neural bases for empathy. Neuroscientist 17, 18-24. doi: 10.1177/1073858410379268

Sheehan, D. V., Lecrubier, Y., Sheehan, K. H., Amorim, P., Janavs, J., Weiller, E., et al. (1998). The mini-international neuropsychiatric interview (MINI): the development and validation of a structured diagnostic psychiatric interview for DSM-IV and ICD-10. J. Clin. Psychiatry 59, 22-33.

Skodol, A. E., Clark, L. A., Bender, D. S., Krueger, R. F., Morey, L. C., Verheul, R., et al. (2011). Proposed changes in personality and personality disorder assessment and diagnosis for DSM-5 Part I: description and rationale. Personal. Disord. 2, 4-22. doi: 10.1037/a0021891

Spielberger, C. H. D., Gorusch, A., Lushane, R., Vagg, P. R., and Jacobs, G. A. (1970). Manual for the State-Trait Anxiety Inventory. Palo Alto, CA: Consulting Psychologists Press.

Strathearn, L., and Kim, S. (2013). Mothers' amygdala response to positive or negative infant affect is modulated by personal relevance. Front. Neurosci. 7:176. doi: 10.3389/fnins.2013.00176

Van Overwalle, F. (2009). Social cognition and the brain: a meta-analysis. Hum. Brain Mapp. 30, 829-858. doi: 10.1002/hbm.20547

Viviani, R., Kächele, H., and Buchheim, A. (2011). Models of change in the psychotherapy of borderline personality disorders. Neuropsychoanalysis 13, 147-160. doi: 10.1080/15294145.2011.10773671

Vrtička, P., Andersson, F., Grandjean, D., Sander, D., and Vuilleumier, P. (2008). Individual attachment style modulates human amygdala and striatum activation during social appraisal. PLoS One 3:e2868. doi: 10.1371/journal. pone. 0002868

Vrtička, P., and Vuilleumier, P. (2012). Neuroscience of human social interactions and adult attachment style. Front. Hum. Neurosci. 6:212. doi: 10.3389/fnhum. 2012.00212

Vuilleumier, P. (2005). How brains beware: neural mechanisms of emotional attention. Trends Cogn. Sci. 9, 585-594. doi: 10.1016/j.tics.2005.10.011

Wittfoth-Schardt, D., Gründing, J., Wittfoth, M., Lanfermann, H., Heinrichs, M., Domes, G., et al. (2012). Oxytocin modulates neural reactivity to children's faces as a function of social salience. Neuropsychopharmacology 37, 1799-1807. doi: $10.1038 /$ npp.2012.47

Conflict of Interest Statement: The authors declare that the research was conducted in the absence of any commercial or financial relationships that could be construed as a potential conflict of interest.

Copyright (C) 2016 Labek, Viviani, Gizewski, Verius and Buchheim. This is an openaccess article distributed under the terms of the Creative Commons Attribution License (CC BY). The use, distribution and reproduction in other forums is permitted, provided the original author(s) or licensor are credited and that the original publication in this journal is cited, in accordance with accepted academic practice. No use, distribution or reproduction is permitted which does not comply with these terms. 